\title{
EDIFICATE
}

I Congreso de Escuelas de Edificación y Arquitectura Técnica de España

València, 4 y 5 de noviembre de 2021

Escuela Técnica Superior de Ingeniería de Edificación

Universitat Politècnica de València

Doi: https://doi.org/10.4995/EDIFICATE2021.2021.13457

\section{Innovación tecnológica de productos de la construcción en las enseñanzas técnicas de la Edificación}

\section{Technological innovation of construction products in the technical education of building}

\author{
María Fernández-Alconchel ${ }^{\mathrm{a}}$, José David Bienvenido-Huertas ${ }^{\mathrm{b}}$ y Juan José Moyano- \\ Campos $^{a}$ \\ ${ }^{a}$ Departamento de Expresión Gráfica e Ingeniería en la Edificación, Escuela Técnica Superior de \\ Ingeniería de Edificación, Universidad de Sevilla, mfalconchel@us.es, jmoyano@us.es b Departamento \\ de Construcciones Arquitectónicas II, Universidad de Sevilla, Sevilla, España, jbienvenido@us.es
}

\begin{abstract}
The generation of new patents and utility models in building is an area of innovation that Technical Architects can cover.

It isn't usual to find Technical Architects who are creators of technological advances in building. At present, thanks to new materials and production technologies in buildings, numerous patents can be generated with great repercussions on the economy and the quality of the built product. It is important to promote this issue in the field of construction, promoting the role of the Technical Architect in the production of technologies.

There are several scientific and technological discoveries that are proof of the need to encourage a professional activity aimed at creation through patents, such as the creation of new systems of implementation, equipment, tools, new materials, among others.
\end{abstract}

Keywords: patents, BIM, teacher education methodologies, construction products, learning

\section{Resumen}

La generación de nuevas patentes y modelos de utilidad en edificación es un ámbito de innovación que pueden abarcar los Arquitectos Técnicos. 
No es habitual que se encuentren Arquitectos Técnicos creadores de avances tecnológicos en edificación. En la actualidad, gracias a los nuevos materiales y tecnologías de producción en edificación, se pueden generar numerosas patentes de gran repercusión en la economía y la calidad del producto construido (Debe et al., 2016). Es importante promover esta cuestión en el ámbito de la edificación, impulsando el protagonismo del Arquitecto Técnico en la producción de tecnologías.

Existen varios descubrimientos científicos y tecnológicos que son la prueba de la necesidad de incentivar una actividad profesional dirigida a la creación mediante patentes, como pueden ser la creación de nuevos sistemas de puesta en obra, equipos, herramientas, nuevos materiales, entre otros.

Palabras clave: patentes, BIM, metodologías docentes, productos de construcción, aprendizaje 


\section{Introducción}

En la actualidad las patentes como elementos de producción e innovación están recibiendo una atención especial en las Escuelas Técnicas Superiores, tanto en las ramas de Ingeniería como de Arquitectura. En este sentido están constituyendo una vía de transferencia del conocimiento, a partir de la revisión de la literatura internacional (Calderón-Martínez, 2014). En algunos países como Estados Unidos e Inglaterra, el marco institucional por excelencia son las universidades.

Las universidades deben cumplir la función de impulsar, ejecutar y apoyar las propuestas para el desarrollo tecnológico de las diferentes empresas de sus países. En ellas, se crea un esquema regulatorio destinado a:

a) Apoyar la creación y afianzamiento de una cultura para patentar las nuevas innovaciones

b) Promover la transferencia tecnológica

c) Apoyar a la mediana y pequeña industria en el desarrollo de competencias tecnológicas

El conjunto de estas tareas debe convertirse en una política universitaria de largo alcance (Del et al., 2009). Las actividades científicas y tecnológicas forman parte de la difusión de los resultados de las investigaciones universitarias. Los resultados de estas actividades deben tener una aplicación para la sociedad y constituir un medio visible de transferencia tecnológica. Por ello, han generado un gran interés desde el punto de vista de la investigación académica. De hecho, la Universidad de Sevilla tiene en su estructura del VII Plan Propio de Investigación y Transferencia ayudas que fomentan la calidad de la protección y títulos de propiedad industrial e intelectual. La financiación llega alcanzar en ocasiones hasta los tres mil euros.

Un enfoque importante es la relación universidad-empresa, en el sentido de que la universidad se incluye entre los agentes que forman parte de una red que transfiere conocimiento y tecnología hacia este sector. La aceleración del cambio tecnológico y el interés por el crecimiento de la investigación han dado como resultado un incremento del desarrollo industrial. Este aumento se puede detectar en el siguiente gráfico (Fig 1) distribuido por comunidades autónomas y en las que puede apreciarse el número de patentes, las 
marcas comerciales y los diseños industriales, siendo la CC.AA. con diferencia la Comunidad de Madrid.

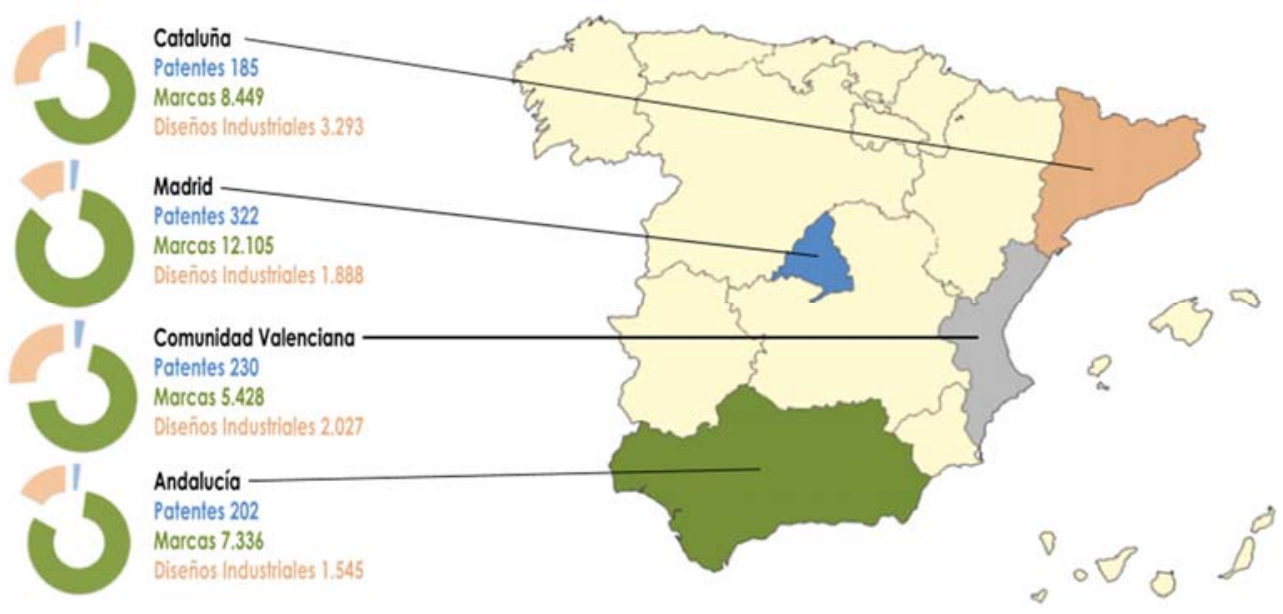

Fuente: OEPM (2020)

Fig. 1 CC.AA. con mayor número de solicitudes de Marcas, Patentes y Diseños Industriales nacionales

\section{Objetivos}

- Implementación del desarrollo de prototipos de patentes en las Escuelas de Ingeniería de Edificación y Arquitectura. Para implementar el desarrollo de prototipos de patentes en todas las escuelas debe de haber una integración entre los grupos de investigación y los trabajos que llevan a cabo los investigadores de la universidad. Debe de existir una base investigadora básica de calidad para que se puede llevar a cabo.

- Impulso de nuevas tecnologías. La utilización de software como BIM, THERM, modelado $3 d$, etc... hacen posible obtener un producto con resultados eficaces y en un periodo corto de tiempo. La utilización de estos softwares para la realización de los prototipos hace que estos tengan mayores niveles de desarrollo y detalles y poder visualizarlos en 3D.

- Implementación de los productos existentes Architecture Engineering and Construction (AEC). El conocimiento de los productos existentes y de la experiencia en el sector de la construcción nos hacen determinar errores y 
deficiencias que tienen algunos productos. Este hecho, nos hace pensar, en prototipos que se pueden realizar para mejorar sus características y eficiencia a la hora de su utilización.

\section{Desarrollo de la innovación}

\subsection{Aplicabilidad de una patente en las enseñanzas técnicas}

En el ámbito del desarrollo de productos de la construcción, quizás el más antiguos y utilizado ha sido el hormigón armado. La primera patente que llega a España de hormigón armado es el sistema Monier, realizada a finales del siglo XIX, concretamente en agosto de 1884. Éste se considera el método más antiguo de construcción con el nuevo material. Con este método se realizaron tuberías, vigas, pavimentos, cubiertas, entre otros objetos. Se ejecutaron a partir de un esqueleto metálico formado por una malla de barras de sección circular que se adapta a la forma del elemento a construir y se protege con revestimiento de cemento.

Como ejemplo de la aplicabilidad de los productos citados y tras realizar una búsqueda de aquellas patentes en el ámbito de la edificación, se han encontrado las siguientes:

- Nuevo elemento de encofrado reutilizable, ligero y económico. Este elemento ha sido desarrollado por el grupo de investigación Materiales y Sistemas Constructivos de la Edificación, perteneciente al Departamento de Edificación y Urbanismo de la Universidad de Alicante (Alicante, 2007).

- Mortero de cal termoaislante, su preparación y su uso en edificación. Universitat de les Illes Balears, 2019 (Patentes - Producción Científica Construcciones Arquitectónicas e Ingeniería de Edificación - Información Del Grupo - Grupos de Investigación - Estructuras - I+D+I - Universitat de Les Illes Balear>, n.d.).

- Estructura prefabrica para construcción mediante sistemas plegables. Universidad Nacional de Colombia. (Estructura Prefabricada Para Construcción Recibe Patente de La SIC - UNIMEDIOS: Universidad Nacional de Colombi>, n.d.) 
El sector de los materiales es uno de los ámbitos donde se desarrolla un incremento de las patentes, con fuertes inversiones apoyadas por proyectos de investigación donde además cuenta con experimentados centros y laboratorios que hacen óptimo la producción de nuevos productos. Sin embargo, a nivel de diseño de prototipos las patentes están más limitadas, porque necesitan de unos componentes de invención que no siempre las universidades son las más adecuadas. Un ejemplo claro es la Escuela Técnica Superior de Ingeniería de Edificación de la Universidad de Sevilla, que cuenta con el grupo de investigación TEP970 (Innovación tecnológica, sistemas de modelado 3D y diagnosis energética en patrimonio y edificación), creado por un grupo de 8 profesores doctores. Tiene por objetivo impulsar la investigación y la transferencia en empresas en el ámbito de la arquitectura e la ingeniería. Presenta evaluación positiva de la Agencia Andaluza del Conocimiento y, ha logrado en apenas unos años ser uno de los grupos referentes a nivel internacional en Modelado 3D del patrimonio histórico mediante técnicas de escaneo y en Análisis de medidas de rehabilitación energética en edificios y patrimonio.

Este grupo de investigación que colabora con el grupo de investigación TEP156 ingeniería gráfica y cartográfica, es uno de los grupos más consolidados en estudios cartográficos, recreación virtual de arqueología, representación del relieve terrestre. El potencial de ambos grupos de investigación supone tener una gran enorme experiencia en trabajos de levantamiento e infografía 3D en excavaciones arqueológicas y patrimonio arquitectónico, lo que se demuestra en los contratos de transferencia tecnológicas de los distintos miembros que componen el equipo. Esta interdisciplinariedad supone un nuevo reto en la conexión entre el desarrollo de la innovación tecnológica de prototipos y la recreación de la construcción de un modelo.

La transversalidad de la investigación del grupo TEP-970 conlleva una relación muy íntima con las Ciencias Naturales, las ciencias tecnológicas y las ciencias en áreas de arqueología de la arquitectura y el patrimonio cultural en general. La creación de plataformas de modelado $3 \mathrm{D}$ de alta precisión es una de las cuestiones más trascendentales en la comunidad científica para la gestión histórica y espacial en el patrimonio cultural. Las técnicas de adquisición de datos precisos requieren de tecnología puntera en equipos de medición. Para la implementación de equipos multidisciplinares especializados en la protección, conservación y difusión del Patrimonio Histórico comprendido distintos campos de conocimiento como la Arqueología, Arquitectura, Conservación, Arte, Historia, Informática y Matemáticas, se hace 
necesario contar con una cantera de equipos disponibles para los investigadores. Trabajar tanto en el ámbito de la nueva planta de la construcción en arquitectura e ingeniería civil, así como en el patrimonio arquitectónico, es abarcar dos facetas para poder crear productos nuevos y desarrollos de patentes.

En el campo de la seguridad y salud en el trabajo, existe un gran número de soluciones que pueden ser útiles para proteger la salud y vida de los trabajadores y prevenir numerosos accidentes de trabajos y enfermedades. Todas ellas se desarrollan en el área del sector de la construcción y la mejora de la eficiencia energética como otro ámbito de explotación. En este sentido el grupo de investigación TEP-970 ha realizado diversas patentes y modelos entre los que destacan el sistema AFF2 en fachada para revestir. La patente diseña un sistema constructivo para evitar el puente térmico en frente de forjados. La disposición de una estructura metálica de barras con un aislamiento fijo adosado a las vigas perimetrales antes del hormigonado hace que el sistema evite la transmisión térmica al forjado. Bienvenido et al.(Bienvenido-Huertas et al., 2018) demostró una reducción del $18 \%$ en la demanda global de energía. El sistema propuesto se describe gráficamente en la figura 2 y mediante un prototipo la fotografía 3 .

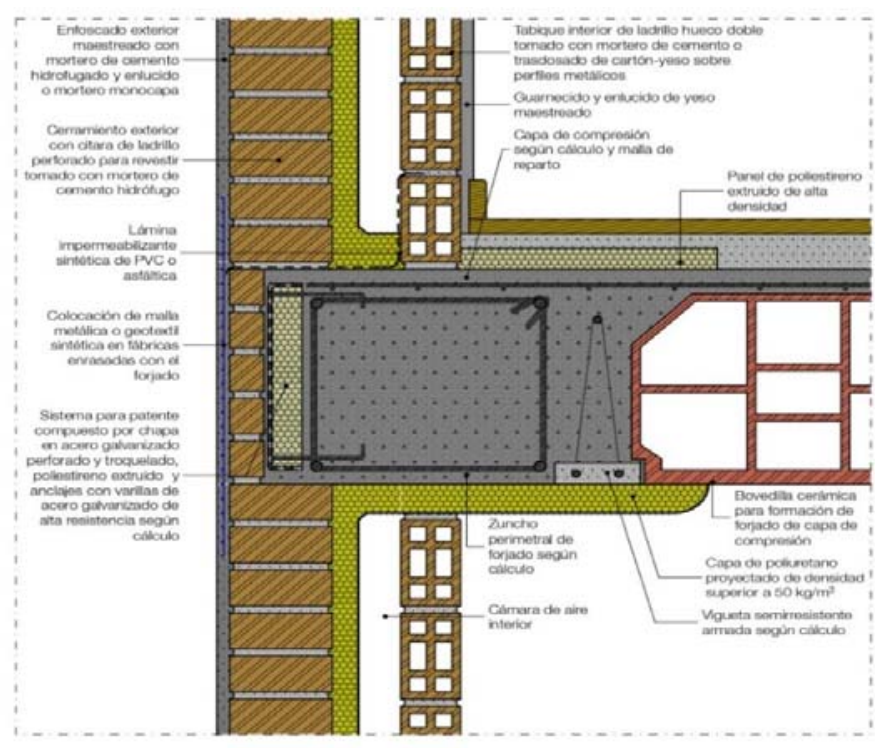

Fuente: Departamento Expresión Gráfica. ETSIE (2013)

Fig. 2 Sistema AFF2 en fachada para revestir 


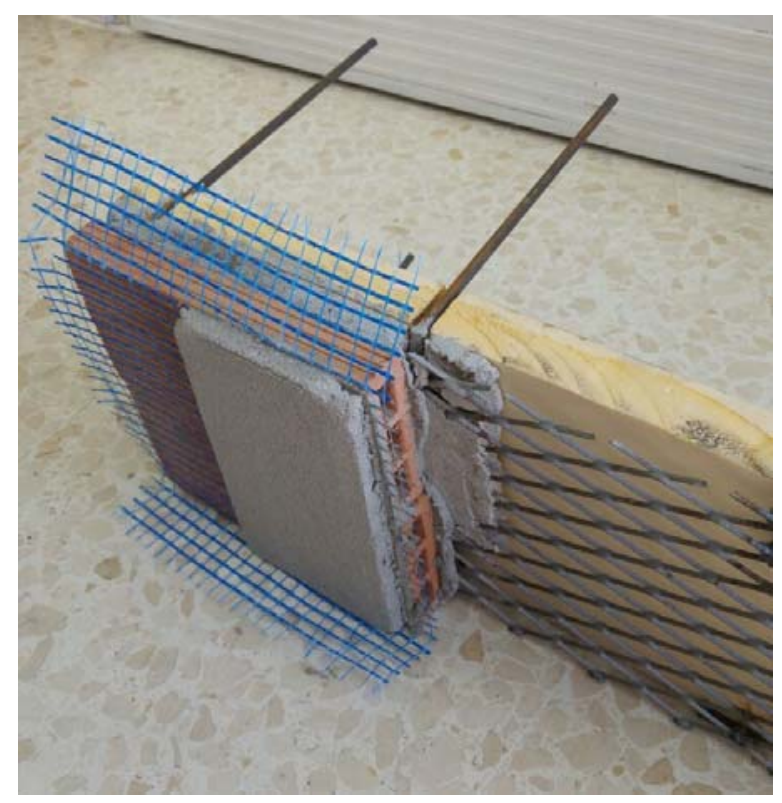

Fuente: Departamento Expresión Gráfica. ETSIE (2013)

Fig. 3 Prototipo sistema AFF2 en fachada para revestir

Para desarrollar la innovación tecnológica en las enseñanzas técnicas de la Edificación, es necesario conocer cuáles son los problemas técnicos que aparecen en el ciclo de vida del proyecto. Aunque existen términos más amplios según los tipos de componentes de la innovación tecnológica (Castro Hermida, 2003). La innovación como significado de crear cosas nuevas y distintas tiene aquí el sentido de creatividad o invención, sean productos fabricados en serie o exprofeso. La naturaleza de la innovación en edificación recae en la invención de productos y procesos. Planear y producir productos nuevos que generen una respuesta a problemas que se generan en el proceso de construcción y de la arquitectura. Para este proceso a nivel educativo tiene que haber un estimulador de problemas y un tutor que dirija estas enseñanzas técnicas. El primer paso es plantear distintas opciones a un problema técnico. Existe un taller de trabajo entre pequeños grupos de alumnos que van discutiendo distintos planteamientos. A continuación, se invita a trabajar en detalles y croquis sobre las diversas soluciones y se fomenta la participación del grupo de trabajo donde el aprendizaje del software es un aliciente para el trabajo innovativo. Diríamos en este sentido que el "gancho" que motiva a los estudiantes en la participación de nuevos productos creativos es la aplicabilidad del software. 
Este trabajo en las enseñanzas técnicas de arquitectura y edificación se está implantando en pequeños grupos de Trabajo Fin de Grado, a un nivel de cuarto curso de carrera en la rama de la arquitectura y la edificación. Concretamente en la Escuela Técnica Superior de Ingeniería de Edificación de la Universidad de Sevilla, el Departamento de Expresión gráfica e Ingeniería en la Edificación está con estas nuevas propuestas de las enseñanzas técnicas.

La dificultad que conlleva compartir docencia entre distintos departamentos limita la transversalidad de los dos grupos de investigación. Pero en la actualidad se está trabajando para poner en marcha una idea de conectividad de competencias para poder explorar a través de trabajos de levantamiento e infografía 3D en excavaciones arqueológicas, patrimonio arquitectónico y nueva construcción, donde las técnicas de adquisición de datos masivos tienen un papel fundamental de recreación en la situación geográfica y la creación de modelos de patentes e innovación de productos y procesos. Esta interdisciplinariedad supone un nuevo reto en la conexión entre el desarrollo de la innovación tecnológica de prototipos y la recreación de la construcción de un modelo. Este sería el gran reto que debemos afrontar a corto plazo.

\subsection{Software de aprendizaje para la funcionalidad de la patente}

Los criterios pedagógicos tradiciones resultan hoy en día de poca eficacia, ya que no motivan a los estudiantes y se han quedado anticuados con las nuevas tecnologías. Por ello, resulta interesante dotar a los estudiantes con nuevos programas informáticos para que puedan desarrollar de una forma más precisa y actual las actividades que quieran desarrollar.

De este modo, la utilización de tecnologías BIM supone un impulso profesional indispensable para poder llevar a cabo todos los proyectos. Esta herramienta de trabajo está implantada en muchas empresas de construcción y arquitectura. Gracias a esta herramienta, los profesionales pueden mejorar la eficiencia y perfeccionar sus trabajos de construcción.

La metodología de trabajo que se utiliza en BIM agiliza y facilita todos los procesos constructivos en el diseño y documentación de edificios (Woo, n.d.). Esto permite que toda la información de construcción, tanto gráfica como no gráfica, la tengamos a nuestra disposición en bases de datos, a la que podemos acceder y recuperar en un momento dado. El resultado nos permite obtener un modelo 3D, en el que podemos representar digitalmente de forma rápida y eficaz nuestro proyecto de construcción. 
Las ventajas de la metodología BIM respecto al trabajo tradicional, son las siguientes:

- Se actualiza automáticamente la información que se edita en cualquier parte del modelo, y ésta directamente se modifica en plantas, alzados, secciones y vistas 3D.

- Se trabaja sobre un único modelo, por tanto, no hay pérdidas de información

- Existe un entorno colaborativo en el que se trabaja de forma coordinada

- Acceso en cualquier momento a toda la información que se requiera

La metodología BIM puede y debe ser utilizada en los años de formación de los alumnos como una herramienta de aprendizaje (Roig et al., 2017). Estas sesiones están basadas en una exposición objetiva de los sistemas, procedimientos y técnicas constructivas de las diferentes fases de obra por parte del docente. La disciplina BIM tiene un carácter práctico que requiere un conocimiento teórico anterior para adquirir las habilidades de representaciones constructivas.

Otro software utilizado es THERM, es el programa informático para el análisis de la transmisión del calor bidimensional a través de elementos constructivos y que permite realizar el cálculo del U-factor, isotermas, vectores de flujo y temperaturas. En la simulación de las patentes, THERM permitió comprobar las mejoras realizadas por las diferentes soluciones constructivas y se obtuvo los resultados de transmitancia térmica de las diferentes patentes (Bienvenido-Huertas et al., 2018).

Existen diferentes softwares para el diseño de productos entre los que podemos destacar dos tipos:

1. Plataformas BIM: se utiliza para crear y organizar datos durante el proceso de diseño, construcción y operaciones. Algunos programas utilizados son: ArchiCAD (Archicad - GRAPHISOF>, n.d.), Revit (Software Revit | Obtener Precios y Comprar Software de Revit 2022 Oficial| Autodesk, n.d.) y Allplan (Software BIM - CAD - 3D - Allplan - ALLPLAN Systems España S.A, n.d.)

2. Programas de modelado: con estos programas se pueden generar escenas, modelar y simular productos. Algunos programas utilizados son: Solidworks (SOLIDWORK>, n.d.), CATIA (CAD Product Design and Engineering Cloud Solutions | Dassault Systèmes, n.d.), Rhino (Rhino - Rhinoceros 3D, n.d.) y Autodesk 
Inventor (Software Inventor | Obtener Precios y Comprar Software Oficial de Inventor 2022 | Autodesk, n.d.)

\section{Resultados}

Los procesos para llevar a cabo las innovaciones tecnológicas han ocasionado un gran aprendizaje hacia nuevos investigadores que forman parte de los grupos de investigación. Parte del proceso en la producción de patentes se relaciona con el tamaño de los grupos de investigación y la capacidad de coordinar el trabajo multidisciplinar. Dependiendo del área de especialidad puede ser más complicado la generación de una patente, aunque si se trabaja de forma multidisciplinar y se dedica tiempo, se obtiene el resultado esperado. Lo cierto es que, muy pocas patentes llegan a ser aceptadas en el ámbito industrial. Un obstáculo puede ser que no hay un vínculo de confianza entre universidad-empresa. Este hecho, nos hace pensar, en prototipos diseñados para tener en cuenta la demanda de las empresas y poder realizarlos para cubrir esas necesidades. Como resultado obtendremos un producto útil y eficaz que sea práctico. Para ello contamos con tres áreas de conocimientos, una es la construcción que nos hace determinar los errores y deficiencias que tienen algunos productos, otra los materiales, que nos muestran sus características y, por último, el conocimiento gráfico en el que disponemos de softwares para realizar prototipos de productos que sean útiles y no tengan la deficiencia que nos encontramos y así mejorarlos para que cumplan con su función.

\section{Conclusiones}

La docencia universitaria está encaminada a la formación de los mejores profesionales en cada escuela. La innovación de impartir clases de BIM en las escuelas esta cada día más presentes debido a la gran demanda en el mundo laboral. Gracias a estos programas, se ahorra tiempo ya que no es necesario convertir desde varios tipos de archivos y se reduce el número de errores al ser menor la necesidad de intercambios de información. Además, estos programas motivan a los alumnos a la hora de realizar los prototipos, ya que cuentan con herramientas en las que se puede diseñar cualquier objeto, pudiendo hacer una vista en $3 d$ de cómo quedaría el prototipo y poder visualizarlo con las texturas de los materiales, cosa que en el método tradicional no se podía hacer. Esto permite, despertar el grado de participación e interés por parte del alumnado. Todo esto no sería posible si las universidades no apoyaran las propuestas para el desarrollo tecnológico. Las patentes están sirviendo como 
modelo de aprendizaje y conocimiento en las escuelas, gracias a ellas, alumnos y profesores investigan sobre un tema y lo llevan a cabo, suponiendo esto, el aprendizaje de programas informáticos, nuevos conocimientos, trabajo en equipo, empleabilidad de nuevos softwares para diseñar y probar los prototipos.

\section{Referencias}

Alicante, U. de. (2007). Listado de patentes. MSCE - Materiales y Sistemas Constructivos de la Edificación. Grupo de Investigación-Materiales y Sistemas Constructivos de La Edificación. https://web.ua.es/es/msce/31-patentes/listado-de-patentes.html

Archicad - GRAPHISOFT. (n.d.). Retrieved June 17, 2021, from https://graphisoft.com/es/solutions/products/archicad

Bienvenido-Huertas, D., Quiñones, J. A. F., Moyano, J., \& Rodríguez-Jiménez, C. E. (2018). Patents analysis of thermal bridges in slab fronts and their effect on energy demand. Energies, 11(9), 2222. https://doi.org/10.3390/en11092222

CAD Product Design and Engineering Cloud Solutions | Dassault Systèmes. (n.d.). Retrieved June 17, 2021, from https://www.3ds.com/es/3dexperience/cloud/catia-product-designand-

experience?utm_medium=cpc\&utm_source=google\&utm_campaign=202101_glo_sea _es_op51508_labl_tt1brand_ewe_exact\&utm_term=catiaexact\&utm_content=search\&gclid=CjOKCQjw5auGBhDEARIsAFyNm9E5TKAepHGm gi9oryzxuQxg5PX8US44A0XJyVE4MKkampXcmzEyLDQaAq1bEALw_wcB

Calderón-Martínez, G. (2014). Patentes en Instituciones de Educación Superior en México. Revista de La Educacion Superior, 43(170), 37-56. https://doi.org/10.1016/j.resu.2014.06.001

Castro Hermida, M. A. (2003). La innovación tecnológica y la gestión de las limitaciones. Innovación Tecnológica En Las Organizaciones, 89-96.

Debe, E. L. A., El, C. E. N., De, C., \& Paralela, F. (2016). Un ámbito idóneo para los arquitectos técnicos. Cercha, 34-35.

Del, M., López Gómez, S., Simón, R. S., Cabrales Gómez, F., \& Tobón, C. G. (2009). The processes involved in a management model for university patents. In AGOSTO DE (Vol. 29, Issue 2). Universidad Nacional de Colombia - Facultad de Ingeniería. 
Estructura prefabricada para construcción recibe patente de la SIC-UNIMEDIOS: Universidad Nacional de Colombia. (n.d.). Retrieved June 14, 2021, from https://agenciadenoticias.unal.edu.co/detalle/article/estructura-prefabricada-paraconstruccion-recibe-patente-de-la-sic.html

Patentes - Producción científica - Construcciones arquitectónicas e ingeniería de edificación - Información del grupo - Grupos de investigación - Estructuras - 1+D+I - Universitat de les Illes Balears. (n.d.). Retrieved June 14, 2021, from https://www.uib.es/es/recerca/estructures/grups/grup/CONSTARQ/produccio/patents/ 2019.html

Rhino - Rhinoceros 3D. (n.d.). Retrieved June 17, 2021, from https://www.rhino3d.com/es/

Roig, R., Antolí, J. M., Blasco, J. E., Lledó, A., \& Pellín, N. (2017). Redes colaborativas en torno a la docencia universitaria. Instituto de Ciencias de La Educación. Universidad de Alicante, 2017, 290-301. https://rua.ua.es/dspace/bitstream/10045/71048/1/Redescolaborativas-en-torno-a-la-docencia-

universitaria_29.pdf\%0Ahttp://rua.ua.es/dspace/handle/10045/70977

Software BIM - CAD - 3D - Allplan - ALLPLAN Systems España S.A. (n.d.). Retrieved June 17, 2021, from https://www.allplan.com/es/

Software Inventor | Obtener precios y comprar software oficial de Inventor 2022 | Autodesk. (n.d.). Retrieved June 17, 2021, from https://www.autodesk.es/products/inventor/overview?term=1-YEAR

Software Revit | Obtener precios y comprar software de Revit 2022 oficial | Autodesk. (n.d.). Retrieved June 17, 2021, from https://www.autodesk.es/products/revit/overview?term=1-YEAR

SOLIDWORKS. (n.d.). Retrieved June 17, 2021, from https://www.solidworks.com/es

Woo, J. H. (n.d.). BIM (Building Information Modeling) and Pedagogical Challenges. 